\title{
Kernos
}

Revue internationale et pluridisciplinaire de religion grecque antique

$20 \mid 2007$

Varia

\section{PAPALEXANDROU Nassos, The Visual Poetics of Power. Warriors, Youths, and Tripods in Early Greece}

\section{François de Polignac}

\section{OpenEdition \\ Journals}

\section{Édition électronique}

URL : https://journals.openedition.org/kernos/331

DOI : 10.4000/kernos.331

ISSN : 2034-7871

\section{Éditeur}

Centre international d'étude de la religion grecque antique

\section{Édition imprimée}

Date de publication : 1 janvier 2007

Pagination : 401-406

ISSN : 0776-3824

Référence électronique

François de Polignac, « PAPaleXandrou Nassos, The Visual Poetics of Power. Warriors, Youths, and Tripods in Early Greece », Kernos [En ligne], 20 | 2007, mis en ligne le 17 mars 2011, consulté le 08 septembre 2022. URL : http://journals.openedition.org/kernos/331 ; DOI : https://doi.org/10.4000/ kernos.331 
substances - in short, the cultural nexus in which $\mu \alpha \gamma \varepsilon i \alpha$ would inscribe itself, had the term already existed.

The third part ("Penser la mageía" 163-216) analyzes how $\mu \alpha \gamma \varepsilon i \alpha$ was practiced and thought, once the complex was perceived as a special field of practices. A lengthy chapter treats fifth century binding spells, centered on the very detailed interpretation of two texts, one from Selinus, the other one from Carystus (163-187); this is compelling, although not entirely novel. C. insists on the vital function of writing not just to fix an otherwise fleeting and transient voice but because the very shape of what is written ("la disposition graphique") has ritual power; this might well be, but we would need more proof than just three underlinings in the Selinuntian lead tablet, and the evidence we have about the connection between reciting a binding formula and writing it down does not bear $\mathrm{C}$. out. The final chapter treats Plato - not just the well-known passages where the philosopher condemns magical practices, but the way Socrates, the master of the powerful word, acts as a sorcerer himself: C. succeeds to show that Plato is well inside the Greek symbolic nexus of magical binding and that to describe him as the ancestor of a rationalist rejection of magic projects later values on him. Surprisingly enough, Plato uses the term $\mu \alpha$ dos only once, and not in the passages where he condemns 'magical' practices but in Rep. 9, 572E where the term denotes the sophists: this again shows that to him the ritual practices were part of the religious tradition, not something alien, and the $\mu \dot{\alpha}$ yos was a master of the word.

C. succeeds in his aim to show that what we call magic was an integral part of the symbolic system of the Greek cities from early on, and he can show how the Persian term was used to think about some of its aspects in fifth century Athens; he is somewhat hazy, however, in explaining why there was a need for such a rethinking. And it goes without saying that his methodological stance makes it impossible to prove or disprove Near Eastern imports into this system: whatever wandering practitioners might have brought (and I am still convinced that they brought a considerable amount of private rituals during the Archaic age), was seamlessly integrated. Scholars who are looking for answers on questions of religious import and cross fertilization, then, have to look elsewhere, as do scholars who want to know more on the Persian specialists that lent their name to the magicians, and on magical practices in early Greece. Scholars, on the other hand, who want to know how Greek reflection on the respective phenomena in their own religion started, will have a useful and well-argued study at their disposition.

Fritz Graf

(The Ohio State University, Columbus $\mathrm{OH}$ )

Papalexandrou Nassos, The Visual Poetics of Power. Warriors, Youths, and Tripods in Early Greece, Lanham / Boulder / New York / Toronto / Oxford, Lexington Books, 2005. 1 vol. $15 \times 22$ cm, 293 p. ISBN : 0-7391-0734-8.

Que ce soit du point de vue de l'histoire de l'art et des techniques, des pratiques sociales ou religieuses, les chaudrons à trépied de bronze occupent depuis longtemps une place importante dans l'étude du monde grec. La forte valeur symbolique de l'objet, comme l'A. le rappelle dans son $1^{\text {er }}$ chapitre, a été mise en valeur pour un certain nombre d'usages et de contextes : objet de don dans le monde des héros de l'épopée, offrande de prestige par excellence dans les grands sanctuaires de l'époque géométrique et du haut archaïsme (IX $\mathrm{e}_{\text {- }}$ $\mathrm{VII}^{\mathrm{e}} \mathrm{s}$.), Olympie en tout premier lieu, prix de concours poétiques ou athlétiques, symbole oraculaire, signe de souveraineté... Elle n'a cependant pas été explorée à fond, en particulier pour les époques les plus anciennes, car les études sur les trépieds ont jusqu'ici négligé une des composantes visuelles majeures de ces objets parfois monumentaux : les figurines d'attache en bronze qui, formant paire, ornaient parfois le haut des poignées ou le rebord de la 
cuve. C'est cette lacune que l'ouvrage de N. Papalexandrou (N.P.) entend combler. Ces figurines ont été jusqu'ici étudiées surtout du point de vue technique et stylistique, pour en distinguer les lieux et méthodes de production, mais sans les constituer en corpus cohérent ni s'interroger sur leur signification : c'est donc sur ces deux points que l'A. concentre son attention.

Bien que l'établissement du corpus (objet essentiel du second chapitre de l'ouvrage, mais approfondi aussi dans les deux suivants) paraisse intéresser en priorité les archéologues, il concerne aussi directement les historiens des religions sur quelques points importants, quand il porte sur les figurines anthropomorphes qui apparaissent vers la fin du IXe s. ou le début du VIII . L'A. en effet ne se contente pas de reprendre les figurines déjà répertoriées comme ornements de trépieds dans différentes publications en raison de la conservation de la pièce d'attache sous les pieds de la statuette, et dont le type le plus courant représente un guerrier au bras droit levé (dans le geste de brandir une lance), fixé au sommet des poignées où il était assez souvent associé à un cheval tenu par une bride. Il élargit considérablement le corpus en prenant aussi en considération des statuettes qui, faute d'attache conservée, ont jusqu'ici été traitées comme des figurines indépendantes, sans rapport avec l'offrande de trépieds. D'un intérêt tout particulier, dans ce sens, est le passage consacré par l'A. aux statuettes d'Olympie au bras droit levé, interprétées depuis Emil Kunze comme des représentations d'un Zeus brandissant le foudre (p. 100-105). Ces figurines ont été souvent rapprochées des statuettes proche-orientales du Bronze récent, dites du «dieu Reshef»ou du «smiting god», images d'un Baal dans lesquelles certains, comme Walter Burkert, ont voulu voir l'origine des figurines « divines » d'Olympie. Procédant à une révision analogue à celle de N. Himmelmann à propos des figurines d'Olympie aux deux bras levés censées représenter une épiphanie de Zeus ${ }^{1}$, N.P. démonte de façon très claire les présupposés à la fois esthétiques et idéologiques sur lesquelles ces interprétations reposaient. Il montre ainsi de manière tout à fait convaincante que ces figurines, comme d'autres de même type trouvées à Delphes, doivent être en fait rattachées à la série des guerriers brandissant une lance et considérées pour cette raison, avec certitude pour certaines qui forment paire, ou avec une forte probabilité pour d'autres, comme des figurines rattachées à des trépieds, parfois accompagnées d'un cheval.

Par effet de dominos, cette démonstration bouleverse toute une série de théories iconographiques et d'interprétations des pratiques d'offrande dans les sanctuaires du haut archaïsme. En premier lieu, l'A. montre la persistance (avec une certaine évolution stylistique) de ce type de figurines dans la $1^{\text {re }}$ moitié du VII ${ }^{\mathrm{e}}$ s., ce qui fournit un indice de la continuité de l'offrande de trépieds à une époque où cette pratique paraît attestée indirectement par différentes sources mais manquer de confirmation archéologique. La lacune intrigante entre les séries bien connues des trépieds géométriques et les offrandes de trépieds connues, à partir de la fin du VII es., par leurs bases et leurs colonnes de support en pierre (les parties métalliques ayant généralement disparu), a suscité la perplexité et laissé penser, par exemple, que les trépieds de tradition géométrique avaient été supplantés pendant un temps, dans la Grèce « orientalisante ", par les chaudrons à protomés orientaux ou s'inspirant de modèles orientaux, ornés de sirènes, têtes de taureaux, lions ou griffons. La continuité de la série des figurines d'attache montre qu'en fait la disparition des trépieds de tradition géométrique n'est qu'apparente et s'explique par un changement des conditions de conservation et non par un changement de pratiques. Du même coup, l'offrande des chaudrons orientaux ou orientalisants apparaît pour ce qu'elle était réellement: un choix, une volonté de se démarquer, de se rattacher à un univers symbolique autre que celui du trépied traditionnel. Cela,

${ }^{1}$ N. HimmelmanN, «Frühe Weihgeschenke in Olympia », in H. Kyrieleis (éd.), Olympia 1875-2000. 125 Jabre Deutsche Ausgrabungen, Mainz, 2002, p. 91-107. 
N.P. l'a bien compris, mais l'interprétation qu'il en donne en expliquant que le bestiaire fantastique qui ornait ces chaudrons exprimait l'idéologie de pouvoirs qui voulaient en imposer par une «rhétorique de la terreur» est un des points particulièrement faibles de l'ouvrage (p. 170-173). À l'instar d'autres objets rares ou évoquant des mondes lointains, ces chaudrons faisaient rejaillir sur leurs détenteurs une aura de prestige et de puissance, reflétant en l'occurrence celle qui émanait des grands empires dont ils provenaient ou étaient censés provenir.

Un autre exemple intéressant des conséquences de l'émergence de ce corpus est le regard que l'on peut porter sur des objets si connus que toute possibilité d'analyse nouvelle semblait épuisée. Tel est le cas de la célèbre statuette de bronze béotienne dite «Apollon de Manticlos ", datée du début du VII ${ }^{\mathrm{e}}$ s. et porteuse de l'une de plus anciennes inscriptions dédicatoires connues. Comme pour les autres figurines, N.P. remet en cause l'identification à une divinité et restitue la statuette en guerrier au bras droit levé, en suggérant de l'inclure dans la série des figurines de trépieds (p. 84-86). Ce dernier point ne peut être définitivement établi, la statuette étant incomplète; on notera cependant que si on l'admet comme hypothèse de travail, on doit restituer (pour des questions de proportion) un trépied de très grande taille, ce qui suppose que la statuette se trouvait facilement à plus de $2 \mathrm{~m}$ ou 2,50 m de hauteur : la lisibilité de l'inscription devient alors problématique, ce qui ouvre le champ à beaucoup de questions. En revanche, l'idée que la statuette serait une représentation de l'idéal à la fois guerrier et juvénile auquel Manticlos s'identifiait, son image sociale en quelque sorte, clarifie beaucoup d'aspects de l'inscription en évacuant l'étrangeté d'une prière à Apollon inscrite sur une figurine qui était censée représenter le dieu.

L'intérêt de la statuette de Manticlos est également la mise en valeur de la beauté masculine juvénile, grâce à une représentation plus élaborée du corps et de la chevelure. Cette mise en valeur permet à l'A. de construire une deuxième catégorie signifiante, celle des figurines de jeunes gens, qui préfigurent les kouroi de la statuaire archaïque. Cette catégorie, qui émerge vers la fin de l'époque géométrique, met en valeur la grâce et la vigueur juvéniles, la charis reçue des dieux, plus que la force exprimée par la lance brandie ou la domination du cheval (p. 166-168). Elle influence la représentation des guerriers attachés au sommet des poignées là où celle-ci se perpétue; mais elle englobe aussi de nouveaux types de figurines, en particulier celles qui, à partir de cette même période, sont attachées sur le rebord de la cuve de part et d'autre des poignées qu'elles soutiennent, formant ainsi des ensembles de doubles paires pour chaque trépied. Ces statuettes très élancées sont souvent associées à des trépieds martelés de grande taille, qui sont considérés comme des productions athéniennes. Certaines de ces paires associant un jeune homme à un personnage à tête de taureau ont de ce fait été interprétées comme une représentation de Thésée et du Minotaure.

La constitution de ce corpus cohérent de figurines, même s'il est assez restreint, est déjà en soi un résultat important des recherches de N.P. mais ne saurait expliquer pourquoi ce livre, avec sa forte composante archéologique, a été publié dans une collection dirigée par Gregory Nagy et dont tous les autres titres portent en conséquence sur la littérature grecque. La raison de ce choix, et du titre de l'ouvrage, ressort des pages d'introduction où l'A. explicite les postulats à partir desquels il propose une interprétation de la signification de ces figurines. Le postulat essentiel, auquel on souscrit volontiers, est que l'image a valeur performative, surtout dans une société où l'écriture ne jouait encore qu'un rôle très marginal : elle appelle un discours, un commentaire, une réponse conçue en fonction du contexte où elle est exposée et joue ainsi un rôle important dans l'élaboration des normes et savoirs partagés. Or le chaudron à trépied, comme l'A. le souligne dans le $1^{\text {er }}$ chapitre, est traditionnellement associé à l'énonciation d'un discours faisant autorité et porteur de vérité : qu'il s'agisse de la parole des rois, du chant de l'aède ou de l'énoncé oraculaire, le trépied signale 
souvent l'autorité du «maître de vérité » tel que Marcel Detienne l'avait dessiné. Tous les arguments avancés par N.P. pour montrer la force de cette articulation ne sont pas également convaincants (avancer, par exemple, p. 16-20, que c'est pour sa "prestation» d'aède qu'Ulysse, au chant XI de l'Odyssée, reçoit en récompense les trépieds des Phéaciens, c'est oublier que le héros s'est auparavant fait reconnaître comme roi, et que c'est en tant que roi qu'il est honoré de cadeaux $)^{2}$, mais l'idée que l'objet appelle et représente en quelque sorte une parole d'autorité, qui pouvait être réactivée à certaines occasions, est essentielle. Elle éclaire bien des aspects des pratiques d'offrande à haute époque : l'apport d'objets de prestige dans des sanctuaires comme Olympie ou Delphes suppose en effet l'existence d'une transmission orale de l'information concernant le dédicant, ses origines, les circonstances de l'offrande, autorisant ainsi la perpétuation et la commémoration ritualisée du kléos. Mais elle explicite aussi le lien avec l'épopée et la chant de l'aède. Les facilités de composition et de mémorisation qu'offrent l'hexamètre et ses formules prêtes à l'emploi aussi bien que l'autorité propre au chant épique constituent en effet de bons arguments pour penser que la transmission et la commémoration prenaient la forme d'une récitation poétique, d'autant plus que les inscriptions dédicatoires ont repris dès l'origine et longtemps gardé la formulation hexamétrique et, comme dans le cas de la statuette de Manticlos, puisé au même répertoire de formules que l'épopée. Les grands trépieds, pour reprendre la formulation de N.P. (p. 3, 109-115), constituaient donc chacun une " performance arena » de commémoration poétique.

C'est dans ce cadre général que l'A. précise les significations attachées aux catégories de figurines qu'il a identifiées. Le cas des guerriers adultes à la lance brandie, les aichmetai de l'épopée, analysé dans le 3 e chapitre, renforce évidemment le message d'autorité du trépied : toute la gestuelle de commandement attachée au maniement de la lance dans l'Iliade montre clairement que la représentation de ces guerriers faisait fonction d'« icône » d'une position de pouvoir destinée à inspirer un respect analogue à celui qui entoure les héros de l'épopée, et à laquelle l'A. donnerait même une dimension sacrée en raison de leur statut de «maitre de vérité » (ce qui parait moins évident).

Beaucoup plus contestable en revanche est l'interprétation que N.P., dans le 4 e chapitre consacré aux kouroi, donne des figurines faisant office de soutiens de poignée. Il construit en effet une opposition systématique entre ces figurines et celles des guerriers hérités de l'époque géométrique : tandis que ces derniers se dressent isolés au sommet des anses, les premières formeraient des groupes impliqués dans une même action collective; les guerriers souligneraient un axe vertical de domination visuelle du haut vers le bas, alors que les soutiens de poignée s’inscriraient dans un schéma horizontal de coordination (p. 162-165)... Toute une série d'arguments sont ainsi avancés pour faire de ce nouveau type de trépieds et de figurines les signes et offrandes de groupes qui mettraient en avant l'identité et l'action collectives, par contraste avec « l'exercice solitaire » d'un pouvoir nimbé de sacré incarné par les guerriers de tradition géométrique. Ces observations, qui paraissent relever de la subjectivité de l'observateur moderne plus que d'une analyse rigoureuse d'un système de représentation, reflètent en fait l'influence avouée des travaux de Ian Morris et en particulier l'application de la formule si malencontreuse d'« idéologie de la cité » que ce dernier avait utilisée pour expliquer les changements de l'Athènes de la $2^{\text {e }}$ moitié du VIII ${ }^{\mathrm{e}} \mathrm{s}$. On se trouve donc devant le syllogisme suivant : les grands trépieds martelés avec figurines de support des poignées sont supposés de fabrication athénienne; or Athènes est la cité où «l'idéologie de la cité » émerge au même moment; donc ces trépieds et leurs figurines reflètent un nouvel idéal où le collectif l'emporte sur l'individuel. Le problème est que la première proposition

${ }^{2}$ Ce point est développé par I. Ratinaud dans son compte rendu de l'ouvrage pour la revue Gä̈a. 
n'est qu'une hypothèse qui, même si elle est exacte, n'empêche pas que des trépieds de ce type aient pu être consacrés par des non Athéniens; et la seconde, qui dénote une vision excessivement simpliste et depuis longtemps dépassée de la cité, suscite l'irritation ou l'affliction quand on voit les travers où peut mener son adoption inconsidérée.

Cette catégorie de figurines appelle donc d'autres observations. En premier lieu, N.P. néglige un facteur technique: avant même d'y rechercher une signification, il faut tenir compte du fait que l'accroissement constant de la taille des trépieds rendait plus aléatoire la stabilité des pièces rapportées de grande taille, comme les poignées; l'apparition de figurines de soutien témoigne de la recherche d'une solution à ce problème ${ }^{3}$. Cela n'exclut pas que les choix iconographiques aient tendu à leur donner une signification. La question essentielle que pose N.P. est en effet de savoir si un changement iconographique de ce genre ne reflète qu'une évolution stylistique, ou bien si l'une et l'autre sont à mettre en relation avec des changements de valeurs dans la société. Mais comme le suggèrent les paires opposant Thésée et le Minotaure (s'il s'agit bien de cela), le face à face des figurines évoque bien plus l'affrontement, la compétition, bref l'agôn, que la coopération : c'est déjà ce que suggère le pied de trépied corinthien du Géométrique récent, trouvé à Olympie et bien connu, où figurent, sur deux panneaux, deux lions affrontés, et plus haut deux guerriers au bras droit levé se disputant un trépied. L'apparition des figurines mettant en valeur la grâce juvénile peut donc bien signifier un changement de paradigme. Mais celui-ci est à rechercher dans une diversification des modèles par lesquels s'effectuent la construction et la «reconnaissance sociale » des élites, pour reprendre les termes d'Alain Duplouy ${ }^{4}$, plutôt que dans la substitution ou l'opposition d'un modèle à un autre : au modèle du guerrier inspiré de l'épopée qui domine au VIII ${ }^{\mathrm{e}}$ s., les figurines de kouroi ajoutent les valeurs d'une charis peutêtre associée à d'autres formes de compétition et de victoire, entre autres athlétiques, donc d'autres formes d'expression de la grâce accordée par les dieux, source de prestige et d'autorité. On rejoint là ce qui a été dit plus haut à propos des chaudrons orientalisants : ces diverses offrandes, et les commémorations poétiques qui les accompagnaient, reflétaient des choix à l'intérieur d'un système élargi de valeurs et de modes de reconnaissance sociale, suggérant une société dynamique et diversifié et non pas un repli ou un immobilisme comme on l'a parfois pensé à propos du VII' $\mathrm{s}$.

L'apparition des figurines juvéniles conduit enfin à une autre interrogation, à laquelle N.P. tente de répondre : comment s'est établi le lien particulier entre Apollon et le trépied, surtout à Delphes ? Cette question est abordée d'emblée dans le 1er chapitre de l'ouvrage, en esquissant des oppositions entre les associations du trépied à Apollon et à Achille dans l'Iliade (p. 25-30), puis à Apollon et à Ulysse dans l'Odyssée au regard de la colonisation et de la fondation des cités (p. 42-47), mais à chaque fois avec des arguments beaucoup trop sommaires et schématiques pour emporter l'adhésion. Le dernier chapitre de l'ouvrage revient sur la question de «l'appropriation du trépied par Apollon». L'importance donnée à juste titre à l'articulation entre trépied et chant poétique, l'efficacité sémantique qu'elle donne au trépied comme source de parole vraie, d'autorité, enfin l'apparition des figurines de kouroi, sont évidemment autant d'indices de la proximité, voire du rapprochement du trépied avec la sphère apollinienne et l'énonciation oraculaire. Mais l'A., en partie en raison des faiblesses relevées dans les chapitres antérieurs, peine à aller beaucoup plus loin que ces constatations générales, et cette difficulté ressort des contradictions auxquelles il est amené avec certaines de ces démonstrations précédentes : ainsi, à rebours de toute la logique qui prévaut dans les premiers chapitres, identifie-t-il à des représentations d'Apollon des figurines ou des

\footnotetext{
${ }^{3}$ Je remercie Francis Croissant d'avoir attiré mon attention sur ce point.

${ }^{4}$ A. Duplour, Le prestige des élites. Recherches sur les modes de reconnaissance sociale en Grèce entre les $X^{e}$ et $V^{e}$ siècles avant J.-C., Paris, 2006.
} 
représentations de figurines sur trépied qu'il avait d'abord considérées comme des images de guerrier ou de kouros (p. 72 et 191; p. 83-84 et 202).

L'ouvrage n'est donc pas exempt de faiblesses et de défauts. Mais il faut lui reconnaittre le mérite de mettre en lumière, autour d'un corpus de bronzes que personne n'avait jusqu'ici identifié comme un ensemble cohérent, un très riche ensemble d'aperçus et de questions touchant aux pratiques sociales et rituelles, à la relation entre productions artistiques et valeurs sociales, à l'articulation entre objets, chant et écriture, qui ouvrent beaucoup de perspectives nouvelles et de réflexions extrêmement stimulantes pour la compréhension de la société grecque de la fin de l'époque géométrique et du haut archaïsme. C'est cette impression de stimulation et d'enrichissement qui domine largement à la fin de la lecture.

François de Polignac

(Centre Louis Gernet [EHESS/CNRS], Paris)

GIANNISI Phoebé, Récits des voies. Chant et cheminement en Grèce archä̈que, Grenoble, Jérôme Millon, 2006. 1 vol. $13,5 \times 21 \mathrm{~cm}, 190$ p. (coll. Horos). ISBN : 2-84137-202-2.

Si vous aimez sortir des sentiers battus ou, plus exactement, revisiter avec un regard neuf des chemins bien connus comme le sont certaines voies sacrées menant à d'illustres sanctuaires, mais aussi les chemins métaphoriques en lesquels se transforment parfois les chants de certains poètes, ne manquez pas de lire cet ouvrage. Il s'agit de la version remaniée d'une thèse de doctorat soutenue, en 1994, à Lyon II (Langues, Histoire et Civilisations des Mondes Anciens) par une diplômée en architecture qui enseigne aujourd'hui à l'École d'Architecture de l'Université de Thessalie (Volos). Le livre devait être préfacé par P. Vidal-Naquet, décédé peu avant la publication; il l'est par J. Svenbro qui fut, lui aussi, membre du jury.

L'idée originale de l'ouvrage, on l'aura deviné, est d'explorer les riches analogies qu'a tissées la Grèce archaïque entre le chemin et le chant, entre la voie et la voix, si l'on préfère. Qu'a-t-on bien pu pressentir de commun à l'époque entre les voies construites et les poèmes ? Pour répondre à la question, l'A. examine tout d'abord, dans une première partie, les voies d'accès menant au temple de quelques grands sanctuaires extra-urbains, celui d'Héra à Samos et ceux d'Apollon à Didymes, à Délos et à Ptôon en Béotie. En ces lieux et en d'autres, les fouilles ont manifesté la présence de nombreuses constructions et sculptures érigées en offrandes, des kouroi notamment; à la différence de celles que l'on trouve aux abords des temples égyptiens, ces offrandes étaient souvent singularisées et propres dès lors à évoquer des souvenirs particuliers et à déclencher des récits dans l'esprit des passants. L'étude des termes utilisés pour désigner ces offrandes (anathèmata, agalmata, sèmata) ainsi que les inscriptions qui y sont gravées témoignent pareillement du but narratif qu'implique leur dépôt en ces endroits. La seconde partie s'attache à montrer les fils qui relient chants et chemins dans la littérature grecque des premiers siècles, à commencer par les emplois des mots oimos et oimè qui véhiculent cette double signification. L'A. consacre aussi un intéressant chapitre à la mnémotechnique antique qui fait du parcours l'essence de la remémoration et l'élément principal des procédés auxquels elle fait appel (cf. les "voies de la mémoire », nous dirions aussi aujourd'hui « les territoires »), elle explore les notions de trace, de pas, de mètre et de rythme, évoque les procédés de la généalogie et du catalogue (sont ici rencontrées des questions qu'abordent aussi les Actes du dernier congrès du CIERGA publiés dans Kernos 19), fait une analyse de deux itinéraires tracés dans l'Odyssée, des divers cheminements que dessine l'Hymne homérique à Apollon et de riches métaphores du chant et du chemin que déploie Pindare. La conclusion élargit plus encore l'éventail en évoquant notamment la thèse anthropologique de la marche humaine considérée comme la source de la production technique, de la langue, de la voix et de la formation d'une mémoire. 